\title{
Wedding Bed Textiles Design Based on Chinese Traditional Auspicious Patterns
}

\author{
Haiqing Miao ${ }^{1}$ Run Wen ${ }^{2, *}$ \\ ${ }^{1}$ College of Art, Soochow University, Suzhou, Jiangsu 215000, China \\ ${ }^{2}$ Key Laboratory of Textile Science \& Technology of Ministry of Education, College of Textiles, Donghua \\ University, Shanghai 201620, China \\ *Corresponding author. Email: dhlg2011@163.com
}

\begin{abstract}
Chinese traditional auspicious patterns are rich in form and imply auspiciousness, which can be used in the design of wedding bedding. But its shape is not simply copied and transplanted, but a redesign of the pattern form. Therefore, it is of great practical value to inherit and innovate the traditional auspicious patterns in China. Through the investigation of wedding market and the analysis of design cases, the author believes that the design of wedding bed textiles based on traditional auspicious patterns requires designers to innovate on the basis of research and inheritance, take its auspicious implication, extend its shape, reconstruct its combination, and Chinese and Western compatibility, so as to make it present a new visual form and keep its vitality.
\end{abstract}

Keywords: Chinese traditional auspicious patterns, Wedding bed textiles design, Auspicious meaning, Pattern form, Red series, Cloud shoulder.

\section{INTRODUCTION}

Chinese traditional auspicious patterns are rich in form and profound in meaning, reflecting the pursuit and yearning of the ancients for a better life. They are the patterns that the Chinese people like to see. They are widely used by designers in industries such as home textiles, clothing, modern design packaging, and interior decoration. Some of these patterns have even become exclusive symbols representing the Chinese nation. "Today's international design platform, expressing nationality is the foundation of design. The design of home textile decorations, which are closely related to people's lives, is no exception. It needs to absorb the nourishment of national culture."[1] In modern wedding bedding products, patterns such as mandarin ducks, dragons and phoenixes, twisted peony, the picture of a hundred kids, etc. often jump into people's eyesight, and they are the main elements in the design of Chinese wedding bed textiles. "Tradition is like roots, and rooted design art can flourish and become stronger, otherwise it will often be a flower in the county and cannot stand the carving of time." [2] ${ }^{70}$ These familiar auspicious patterns are the root of Chinese wedding bed textiles design. Therefore, their new characteristics in the new period are worthy of analysis and research. The article hopes that through its innovative application research in the wedding bed textiles design, it can summarize its characteristics, and inspire designers to continuously explore and innovate the application of traditional Chinese auspicious patterns to form a unique Chinese style of wedding products.

\section{THE CONNOTATION AND ELEMENTS OF CHINESE TRADITIONAL AUSPICIOUS PATTERNS}

The concept of "auspiciousness" appeared relatively early. On the unearthed oracle bones, there are inscriptions such as "吉 (good luck)" and " 大吉 (great good luck)". The ancients believed that auspiciousness was a sign and thing such as auspicious propitiousness, good luck and good fortune. They placed their prayers for auspiciousness on patterns of animals, plants, utensils, geometry, characters, numbers, etc., and presented them through various carriers such as painting, carving, embroidery, weaving and other 
methods. People usually call these patterns as auspicious patterns. "The variety of traditional Chinese auspicious patterns is abundant, and they have evolved with different historical periods, reflecting the ancestors' continuous pursuit of auspicious and beautiful life. Through the use of symbolism, meaning, homophony, table number, text, and comprehensive use of other artistic techniques, the ancients created countless elegant and popular decorative art images."[3] These patterns mainly include themes such as peace and longevity, having many children and good fortune, high positions in office and good fortune, joy and prosperity, etc. For example, the mandarin duck symbolizes love and loyalty, the deer and crane symbolize longevity, and the peony symbolizes wealth and honor. The homophonic sounds include the 考(猫 mao, cat)耊(蝶 die, butterfly)老人(means the elderly of more than 80 years old, 喜(喜鹊 xique, magpie) 上眉 (梅 mei, plum) 梢 (means happiness appears on the eyebrows), and 三阳(羊 yang, goat)开 泰(means auspicious beginning of a new year). The superfacial symbols include patterns such as the Dark Eight Immortals, Ruyi, and the Eight Auspiciousness, and the text has patterns such as 福 (fu, means blessing) 禄 (lu, means fortune) 寿(shou, means longevity) 喜 (xi, means happiness). Most of these patterns are represented by concrete and image, and they are simple and complicated in form, some are single patterns, and some appear in combination. Monolithic patterns represent by a certain kind of animal, plant, object, etc., used alone to express auspicious meaning. For example, tortoise symbolizes longevity, pomegranate symbolizes multiple children, bat symbolizes multiple blessings and so on. Combination use means to use two or more elements. For example, the combination of longevity peach, bergamot, and pomegranate represents multiple children and longevity, the combination of cat and butterfly represents the longevity of old people, and the combination of dragon and phoenix implies auspiciousness and so on. Studying the connotation of these patterns will help home textile designers to rationally and creatively use these patterns according to the design theme in the current design.

\section{TYPICAL CHINESE TRADITIONAL AUSPICIOUS PATTERNS AND MODERN WEDDING BED TEXTILES DESIGN}

The wedding night in the bridal chamber is one of the four great joys of life. Marriage has been a great event in life since ancient times, and wedding celebration is also an important moment in life. "However, there are two most prominent celebrations in the folks: the first is the marriage of men and women. The second is to give birth to children. The ancients valued the continuation and prosperity of the family. Don't cut off the incense, otherwise you will be unfilial."[4]368 People wish the newlyweds an early birth, promotion, wealth, marriage, peace and happiness, etc., and these blessings are placed on marriage ceremonies and wedding supplies. In today's society, about 10 million couples get married every year, there is a great demand for wedding products, and they are willing to spend a lot of money to decorate new houses. The layout of the Chinese-style wedding room is still the mainstream, and the big red wedding bedding with traditional auspicious patterns is the first choice for the layout of the Chinese-style wedding room. There are many traditional Chinese auspicious patterns, which have been mentioned before, so the author won't repeat them here. Among them, many patterns are used by designers in the design of wedding bedding. Commonly used are pictures of a hundred kids, pomegranates, melons and tangled branches, which means blessing the new couple to have many children. Patterns of blessings of wealth and auspicious include as dragons and phoenix, peony, eight auspicious, ruyi, and Baoxiang flowers. Patterns of blessings of fortune and peace include bats, gourds, deer, sea and river cliff patterns. Patterns of blessing marriage and harmony include mandarin ducks, double 喜 characters, double lotus, magpies on the plum branches and other patterns. Patterns of blessing longevity include longevity peaches, deer, ganoderma, longevity flowers and other patterns. However, the wedding bed textiles design is inseparable from innovation, but innovation is not a simple copy and transplant. "The most important thing to find the local design language in the tradition is to reposition the tradition in contemporary culture on the basis of understanding to fit the theme of the design, and to understand and express it with the minds of modern people. It is necessary for the designers to expand, 
transform and apply the traditional features of the native language and the opportunities of the times to make it rich in the characteristics of the times."[2] ${ }^{72}$ Designers must have a new understanding, research and performance of auspicious patterns in order to demonstrate the cultural personality of home textile products.

\section{THE INNOVATIVE APPLICATION OF CHINESE TRADITIONAL AUSPICIOUS PATTERNS IN THE WEDDING BED TEXTILES DESIGN}

In the design of modern wedding bed textiles, auspiciousness is one of the important themes. The author found through market research that consumers are willing to spend a high price to purchase wedding bedding to decorate new houses. The mainstream home textile brands in the market will specially develop wedding series bed textiles. The bed textile patterns are mostly traditional themes, and the color is mainly in red, using printing, jacquard plus embroidery, stitching, knitting and other production and decoration techniques. These characteristics are worthy of people's research and summary.

\subsection{Conveying the Auspicious Meaning of Patterns}

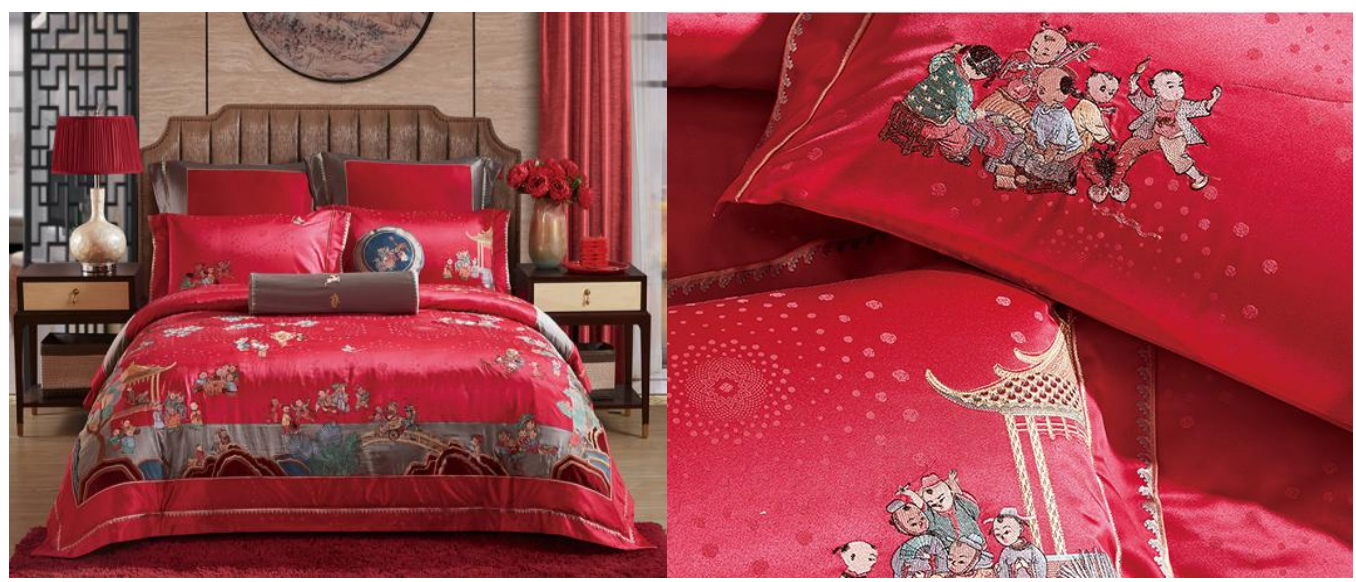

Figure 1 Wedding bed textiles with the picture of a hundred kids.
The implied meaning of traditional auspicious patterns has always been the key to people's love and even fascination with it. In the wedding bed textiles design, designers will use those classic auspicious patterns to express the wedding auspicious theme, and use familiar auspicious patterns to create festive product styles to create a sense of wedding ceremony, and convey a strong auspicious meaning to attract consumers to buy. "Since ancient times, in the wedding dowry, there is a quilt with a brocade quilt like Baizi's picture, which is used to contain celebrations and blessings. At the same time, I wish the bride a son early, a full house, family and beauty." [5] "Figure 1" shows the home textiles and bedding "A Hundred Kids to Bring Fortune". The boys on the quilt are frolicking, flying kites, carrying lanterns, which are with various shapes, lifelike, among them carrying pomegranate has the meaning of having many children, playing with the horse represents the success, holding ingots has the meaning of bring fortune to this family. The designers use the implied meaning of the pattern to bless the newcomer to give birth to a precious child early, and to have many children and to be happy and fortunate. The products are stylish, elegant and luxurious, with a strong wedding atmosphere. "Figure 2" shows the wedding developed by Luolai Home Textiles 


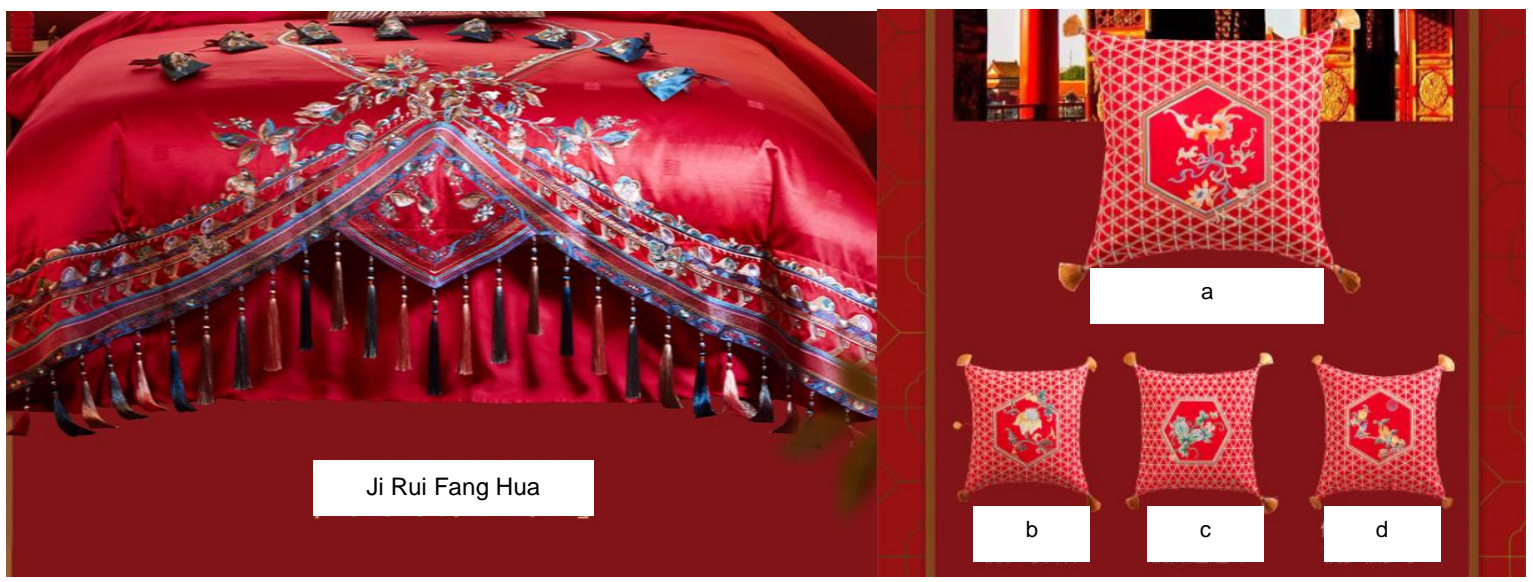

Figure 2 Wedding bed textiles of Luolai Home Textiles.

a Bat/gourd to bless for fortune, peace and health

In the product "Ji Rui Fang Hua", the designer uses the hall ridge of the Forbidden City as the quilt cover decoration. The dragon and the phoenix are lingering each other, the tassels hang down like a phoenix crown, and the auspicious eight-treasure bags are hung on both sides of the bed as decorations. On the wedding day, they are equipped with longan, red dates, peanuts, and lotus seeds to wish the couple an early birth. The pillows are inspired by the pane elements of the Forbidden City. Combined with patterns that symbolize longevity (longevity flowers and Ganoderma lucidum), melons (melon and butterflies), happiness, fortune, peace, and health (bats and gourds), and festive multi-fortune (bergamot and magpie), the wedding theme is vividly highlighted, and all convey the meaning of longevity and good fortune, many children and many blessings. "Pomegranate, melon, and gourd, these three plants, some of their traits, some of the same pronunciation, not only in the meaning of the marriage and childbirth are related to the celebration, but also to be passed on from generation to generation." $[4]^{381}$ Therefore, the combination of traditional auspicious patterns and modern wedding bedding products not only makes the product rich in cultural connotations, it is the extension of traditional wedding concepts in the new era, but can also create high-end solemn wedding products and form a distinctive and novel oriental design style.

\subsection{Combination of Chinese and Western patterns}

In the wedding bed textiles design, the combination of Chinese and Western patterns refers to the combination of different Chinese and Western patterns, which are perfectly integrated through the unity of colors, crafts and materials. There are also original patterns as the skeleton, and other patterns are grafted onto it. "Figure 3" shows the application of double happiness pattern grafting. The main pattern on the picture is a foreign pattern at a glance. The basic skeleton is composed of European curly grass pattern and round lace. The auspicious text round-喜 in the center is obviously grafted. "Figure 4" shows an example of the combination of paisley and dragon and phoenix patterns. "The city of Paisley in southwestern Scotland, England, was famous for producing wool shawls and turbans in the 19th century. People named a pattern commonly used on shawls after the city's name, which is the Paisley pattern. This kind of pattern has formed a kind of everlasting flower style genre in dyeing and weaving art design." It is characterized by an oval shape at one end and a round tail with a slender curly grass shape at the other end. It is slender and dexterous. It is often filled with flowers. It is widely used in European interior furnishings, clothing, and textiles, and has become a classic European pattern element, which is also called "ham pattern". The designer is very bold, combining the auspicious dragon and phoenix pattern with the elegant and romantic Paisley pattern, combining the two on the duvet cover 
under a unified color tone. The bed products are festive and fashionable. "Figure 5" shows the combination of the corner pattern on the pillow with the sea water pattern, pavilion and the auspicious cloud pattern. Because the sea water pattern and the curl grass pattern have the characteristics of curling, the combination of patterns and shapes is still suitable. It's just that the color matching should be echoed, it seems to be more coordinated and unified, and it can better express the theme of 千里共婵娟 (meaning: friends' hearts are always together). Both grafting and combination are innovative attempts to apply auspicious patterns in the new era.

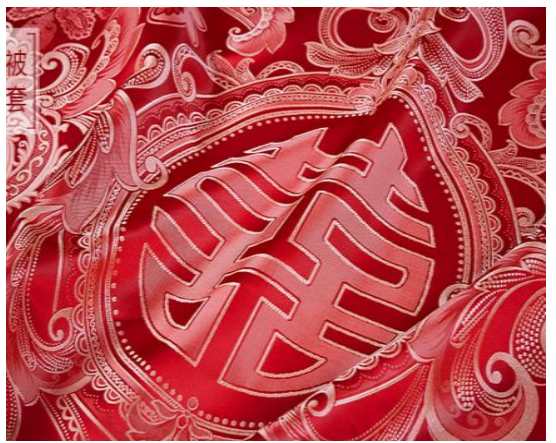

Figure 3 Application of double-喜 grafting.

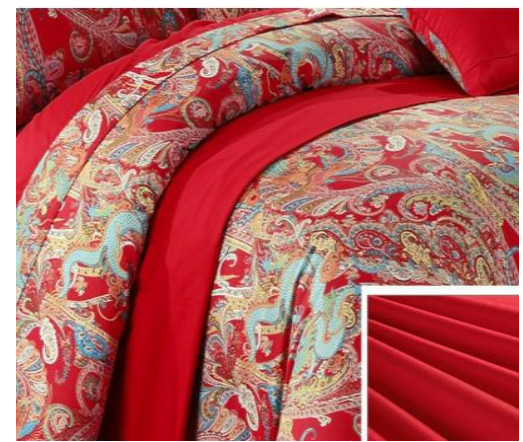

Figure 4 The combination of dragon and Paisley pattern.

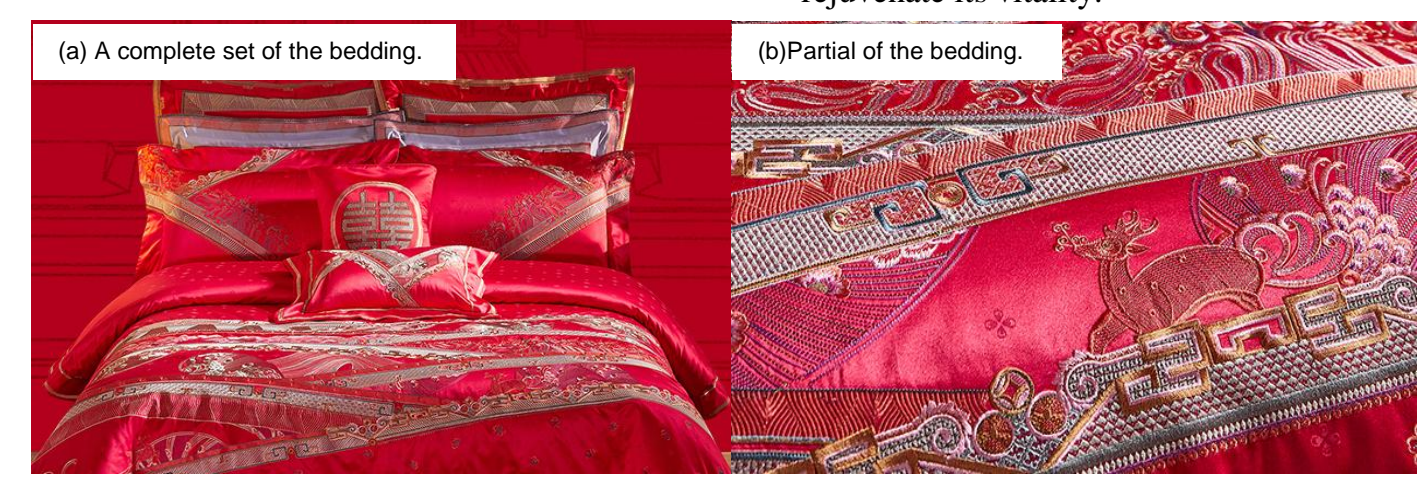

Figure 6 Wedding bedding with patterns such as city walls, belts of court dress.

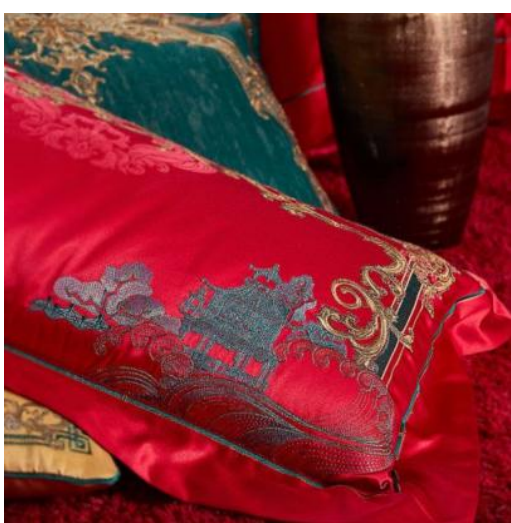

Figure 5 The combination of seawater, pavilion, etc. and curly pattern.

\subsection{Reconstruction of Pattern Modeling and Decoration Matching}

The reconstruction of bedding patterns is to extract the pattern elements, break them up and disassemble them, and make them present a new form through a certain method of combination and arrangement. It is one of the methods of modern textile decoration design. "Figure 6" shows the extraction and combination of the patterns on the walls of the Forbidden City, belts of court dress, and porcelain, the repeated interlacing and overlapping edges and patterns, using reconstructed modern decoration techniques to deconstruct and splice to bring the perfect presentation of tradition and modernity. Auspicious patterns such as unicorns, deer, coins, river cliffs and mountains are distributed among them. The meaning of blessed life without borders and extraordinary wealth is clear at a glance. Reconstruction has brought them new life. But reconstruction is not simply a combination of permutation and superposition, it needs to establish the skeleton structure according to the basic type, and then recombine it to rejuvenate its vitality. 
There are six-piece, eight-piece, ten-piece and other combinations for wedding bedding, consisting of quilt cover, bed sheet (cover), middle pillowcase, cushion, envelope pillowcase, etc., mostly printed and embroidered on jacquard fabrics. The printing and jacquard patterns are the base plate, the color is mostly red, a small part is pink, some are decorated on the floor, some are decorated with clear ground, some are abstract geometric patterns, some are realistic patterns of concrete flowers, and some are decorated with concrete patterns. Embroidered patterns play a role in highlighting the theme, and are often decorated at the front end (the bedside part) and corners of the duvet cover, which contrast with the big red color of the base. However, the design and use of these elements do not need to deal with the integrity and compatibility of the whole set of bedding, that is, the matching design of interior textile decorations; "The matching design of interior textile decorations refers to the unified decorative tone of the overall environment. The supporting artistic means make indoor textile decorations form a specific style of spatial artistic expression, which is the re-creation of the indoor environment."[7] The designers should properly handle the relationship between the theme style and pattern shape, pattern composition, color, and craft materials. The A-version pattern of the quilt cover in the wedding bedding is the main part of the design. The pattern on the middle pillowcase follows the A-version pattern design, which will be simplified, changed size, line thickness, density, etc., or part of the decoration may be extracted. The patterns of envelope pillowcases and cushion covers follow the B version (quilt lining and bed sheet) design, and the decoration is usually relatively simple. This design saves development and production costs, and also achieves a unity of matching from the four aspects of shape, material, quality, and color, presenting an integrated visual form with distinct primary and secondary, and different densities. From "Figure 1" and "Figure 6", the readers can get a glimpse.

\subsection{Red Is the Main Hue}

Color is the clothes of the product, which gives consumers the most intuitive feeling, and is also an important element to express the style of the product. At the moment, bright red is the symbolic color of the Chinese nation. It contains connotations such as auspiciousness, warding off evil spirits, and nobility. It is the color of good fortune and is used in weddings, New Years, and major festivals. However, in different periods of ancient times, the auspicious color was not all red. "The weddings people saw today were mostly red for auspiciousness. However, from the pre-Qin period to the Sui and Tang dynasties, black was firstly regarded as the auspicious color, and then green was regarded as the auspicious color. The color of red was auspicious in the Ming and Qing Dynasties and continues to this day. "[8] The wedding bedding products developed based on auspicious patterns are mostly red, and the patterns are mostly red, yellow, blue and other colors. There are also graphics and color schemes that take example by Ming and Qing embroidery costumes, folklore New Year pictures, architectural elements, and paintings. It is necessary to create a festive atmosphere, but also to express the exquisiteness of the product, with uniform colors and contrasts. As one of the contents of the form, the overall color matching of the bedding should also be matched and echoed with each other. In "Figure 7", the main body bright red is used as the color matching of the dark blue pillow's edging and the decorative piping of the pillow and echoes it. The dark blue is also used as the edging color of the cushion, pillow and duvet cover, which they assist each other. In short, the color matching of wedding bedding with Chinese auspicious patterns is mainly red. Of course, in addition to red, the pink color scheme is fashionable and romantic, but it accounts for a small proportion. The pattern is concentrated on peony, lily and other patterns. Few embroidered decorations, mostly jacquard quilt covers, quilt covers, sheets and pillowcases, combined with lace decorations, and combined with ribbons, crystal yarns and other materials to create a stylish and romantic wedding space. 


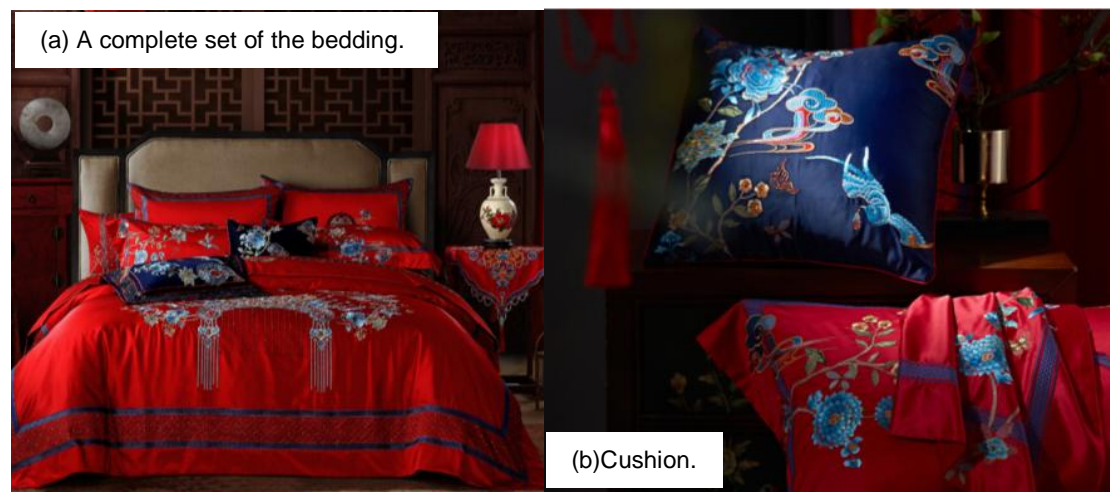

Figure 7 Bright red wedding bed textiles.

\subsection{Combination Application of Patterns and Cloud Shoulder Style}

"Cloud Shoulder, a female red artwork that was used by women as shoulder collar decorations from the Sui and Tang Dynasties to the Republic of China, It is like colorful cloud is on their shoulders, called 'Yunjian (cloud shoulder)'." [9] In the Qing Dynasty, cloud shoulder was a must-have dress for Han women in marriage." Behind the exquisite craftsmanship of cloud shoulder implied the selfesteem of unmarried women and the expectation of love. The skill of threading needles has become the standard for judging a girl, and even determines her future marriage and family." [10] It is very popular to use it with the big red dress in folk weddings. However, this marriage custom has revived, becoming popular in contemporary Chinese weddings, and then being researched and innovatively applied to the design and development of wedding bed textiles by home textile designers. However, the shape of the cloud shoulder is complicated and should not be copied in its entirety. According to the style of the bedding, the designer extracts a part of its shape, makes corresponding simplifications, and then uses it for the decoration of the bedding. Cloud shoulder styles are often decorated on quilt covers and pillowcases. The decorative shapes on the two wedding bedding products in "Figure 8" and "Figure 9" are obviously borrowed from the style of cloud shoulders, and part of the shape is extracted. One is decorated on the quilt cover, and the Jiangya sea water and phoenix pattern are organized in the contour line of the shape, decorated and running through both sides of the quilt cover. The other is decorated on the four corners of the quilt cover, one corner of the pillowcase, and one side of the small pillowcase, embroidered with a fish-carrying boy pattern, decorated with long knots and tassels, which symbolizes prosperity and good luck. In short, the application of traditional clothing cloud shoulder styles enriches the styles of wedding bedding and further highlights the theme of wedding bedding.

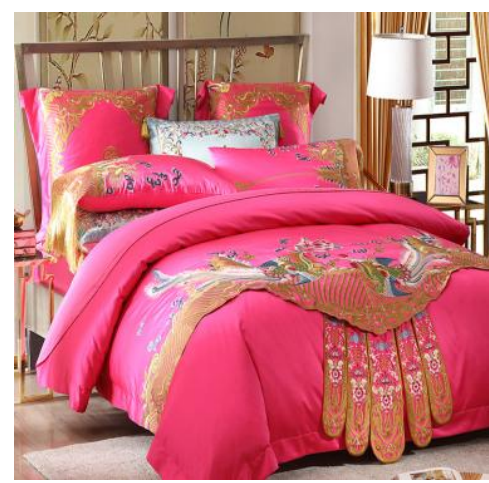

Figure 8 Cloud shoulder on quilt cover.

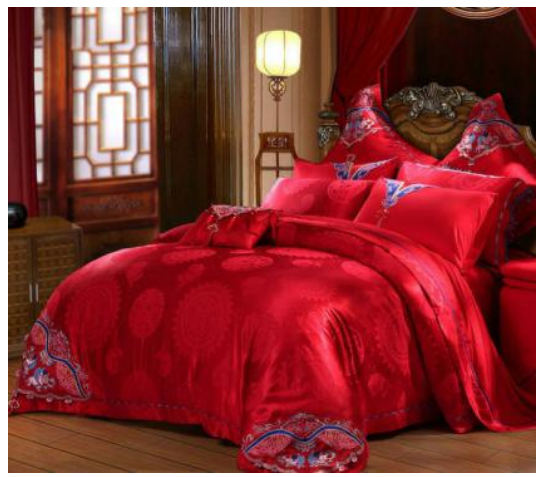

Figure 9 Cloud shoulder on bedding angle and pillowcase angle.

\section{CONCLUSION}

Chinese traditional auspicious patterns are the most splendid part of Chinese national culture, with diverse shapes and rich auspicious meanings. The implied meaning is in line with the current consumers' expectation for a happy married life. In the design of modern wedding bedding, designers 
have the responsibility to study the connotation of traditional Chinese auspicious patterns, innovate and apply them on the basis of inheritance, and abandon simple pattern stacking and ethics. They must reconstruct and combine the form according to the modern design language to be compatible with China and the West. At the same time, they should do a good job in the matching design of the bedding, so that the shape can continue to be extended in the wedding home textile products, and the auspicious meaning continues to be extended. It is a necessity to establish the cultural selfconfidence of the Chinese nation and design fashionable Chinese-style wedding products.

\section{AUTHORS' CONTRIBUTIONS}

Haiqing Miao wrote the manuscript, while Run Wen contributed to revising and editing.

\section{REFERENCES}

[1] Xu Baijia, National Pattern Element Is the Soul of Modern Design - Innovation of Traditional Pattern of Miao Nationality in Guizhou in Modern Domestic Textile [J]. Journal of Nanjing Arts Institute (Fine Arts \& Design), 2011(4): 114. (in Chinese)

[2] Hang Jian, He Jie, Jin Daiqiang. Sui Han San You.Chinese Traditional Graphics and Modern Visual Design [M]. Jinan: Shandong Pictorial Publishing House, 2005. (in Chinese)

[3] Miao Haiqing. The Study on the Application of Chinese Favonian Patterns in Modern Hometextile [D]. Suzhou: Soochow University, 2008: 3. (in Chinese)

[4] Zhang Daoyi. Study on Auspicious Culture [M]. Chongqing: Chongqing University press, 2011. (in Chinese)

[5] Du Hengwei. The Style of Hundred Child Graph in the Qing dynasty Palace [J]. Collectors, 2018(4): 45. (in Chinese)

[6] Xu Baijia, Tentaive Study on PAISLEY Pattern [J]. Art \& Design, 2003(8): 34. (in Chinese)

[7] Huang Guosong, etc. Fabric Pattern Design Advanced Textbooks [M]. Shanghai: Shanghai People's Fine Arts Publishing House, 2005: 94. (in Chinese)
[8] Zhuge Kai. Red Symbol of China [J]. Journal of Suzhou Art \& Design Technology Institute, 2003(3): 20. (in Chinese)

[9] Liang Hui'e, Xing Le, Chinese Traditional Folk Dress Adornments: Yunjian Culture Research [M]. Zhengzhou: He'nan Literature \& Art Publishing House, 2013:18. (in Chinese)

[10] Deng Xiangpeng. From the Divine Worship to the Common Customs - History of Art Development About Cloud Shoulder [J]. Art \& Design Research, 2016(1): 43. (in Chinese) 